\title{
Dependence of silicon position-detector bandwidth on wavelength, power, and bias
}

\author{
J. H. G. Huisstede, B. D. van Rooijen, K. O. van der Werf, M. L. Bennink, and V. Subramaniam \\ Biophysical Engineering and MESA + Institute for Nanotechnology, Department of Science and Technology, \\ University of Twente, P.O. Box 217, 7500 AE Enschede, The Netherlands
}

Received August 16, 2005; accepted October 18, 2005

\begin{abstract}
We have developed a two-LED wobbler system to generate the spatial displacement of total light intensity on a detector surface, facilitating the acquisition of frequency responses up to $600 \mathrm{kHz}$ with high accuracy. We have used this setup to characterize the low-pass filtering behavior of silicon-based position detectors for wavelengths above $850 \mathrm{~nm}$ by acquiring the frequency responses of several quadrant detectors and positionsensitive detectors as functions of wavelength, applied bias voltage, and total light power. We observed an increase in bandwidth for an increase in applied bias voltage and incident-light intensity. The combined effect of these parameters is strongly dependent on the detector used and has significant implications for the use of these detectors in scanning probe and optical tweezers applications. (C) 2006 Optical Society of America OCIS codes: $040.5160,040.6040,230.5160,230.0250$.
\end{abstract}

In atomic-force microscopy (AFM) and optical tweezers (OT) the optical beam deflection method is used to accurately measure the position of the probe. For both techniques the power spectral density of the deflection signal of the probe (i.e., AFM tip or bead) is widely used to calibrate the stiffness of the probe. ${ }^{1,2}$ For these applications it is important to obtain the frequency response of the detector and other electronics to be able to apply appropriate corrections if necessary. The Brownian motion of the probe is a result of the thermal activity of the surrounding medium and can be considered white noise. ${ }^{3}$ Therefore the power spectral density directly reflects the transfer function of the probe, from which the stiffness of the probe can be determined. In OT applications in which a $1064 \mathrm{~nm}$ laser is often used it was noticed that the frequency response was unexpectedly attenuated above $\sim 5 \mathrm{kHz}$, caused by a low-pass effect of the silicon position detector, ${ }^{4}$ resulting in an incorrect determination of the stiffness.

The electrical response of a typical silicon photodiode to infrared light consists of both a fast and a slow component. The fast component results from optical absorption in the depletion layer, whereas the slow component results from absorption beyond this layer owing to the transparency of silicon in the near infrared. In the depletion layer the electron-hole pairs created are rapidly swept to the electrodes, whereas electron-hole pairs created outside the depletion layer first diffuse into the depletion region before going to the electrodes. As a result the bandwidth of the detector is limited dramatically. ${ }^{4}$ For OT, although several groups of researchers have investigated this phenomenon, ${ }^{4-6}$ the experimental configurations used had inherent limitations and the low-pass effect was shown only for a limited bandwidth measured indirectly or for only one wavelength. Using our custom-designed LED wobbler, we have been able to directly measure the frequency response of a selection of position detectors with high accuracy and for several wavelengths.

We investigated two types of silicon position detector: the quadrant detector (QD) and the position- sensitive detector (lateral effect diode, PSD) as a function of wavelength, applied bias voltage, and total incident light power. A QD works by measuring the difference in light power incident on its four quadrants. A PSD measures the center of energy of a spot. Besides the difference in method of detection, the deflection sensitivity of a PSD is insensitive to spot size, which is not the case for a QD. Furthermore the detection range and the deflection sensitivity of a QD can be restricted more by the shape and size of the spot than can those of a PSD. ${ }^{7}$ A spatial deflection of light power has to be generated for the frequency response of the detectors to be determined. For this purpose we developed a two-LED wobbling system (the LED wobbler) as depicted in Fig. 1. With this system a spatial deflection of light power is simulated, analogous to the experimental situation in AFM and OT. Each of the two LEDs is frequency modulated above the LED's threshold current level, one with $\sin (\omega t)$ and the other with $-\sin (\omega t)$, result- (a)

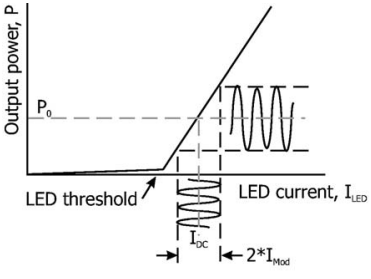

(c)

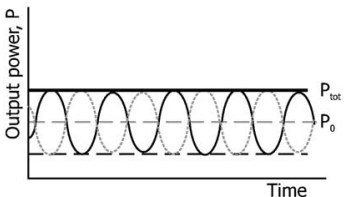

(b)

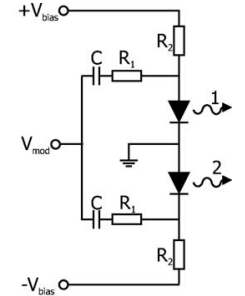

(d)

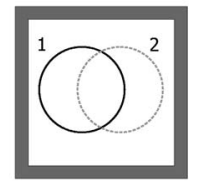

Fig. 1. (a) Principle of frequency modulation of a LED. (b) Schematic of the LED-wobbler circuit used to superimpose the frequency modulation on the dc current: $R_{1}=50 \Omega, R_{2}$ $=1 \mathrm{k} \Omega, C=100 \mu \mathrm{F}$. (c) Light intensity of both LEDs in time. The phase difference between the two LEDs is $180^{\circ}$. The darker curve corresponds to LED1; the lighter curve, to LED2. (d) Schematic drawing of the light impinging on a detector. The solid circle corresponds to LED1; the dotted circle, to LED2. 
ing in a $180^{\circ}$ phase difference [Fig. 1(a)]. The LED wobbler allows us to measure the frequency response of both QDs and PSDs up to high frequencies with high accuracy in a simple way. A modulation depth in the deflection signal of $10 \%$ of the total light intensity was used. The resultant variation in total intensity was found to be less than $0.5 \%$, showing a good match in characteristics of the two LEDs used. We used LEDs in the range 405-1070 nm (Roithner Lasertechnik LEDs; 405, 505, 605, 700, 830, 850, 910, 970, and $1070 \mathrm{~nm}$ ). All LEDs except the 910 and 970 $\mathrm{nm}$ LEDs had a response time of $\leqslant 25 \mathrm{~ns}$, resulting in a bandwidth of at least $6 \mathrm{MHz}$. The response time for the 910 and $970 \mathrm{~nm}$ LEDs was approximately $1 \mu \mathrm{s}$, resulting in a bandwidth of $\sim 150 \mathrm{kHz}$. The frequency responses of the detectors at these two wavelengths were corrected for the transfer function of the individual LEDs, determined by use of a UDT-PIN H040 (UDT) photodiode with a bandwidth of $>1 \mathrm{MHz}$. The custom-built electronics used to acquire the detector signals were optimized to suppress gain peaking. Frequency responses were acquired with a gainphase analyzer (Hewlett-Packard HP 4194A) and transferred to a PC using custom LabView software. The frequency responses shown here were corrected for the response of the electronic hardware.

We first investigated the low-pass behavior of a silicon position detector as a function of wavelength. The typical frequency response of a standard QD operating at no bias is shown in Fig. 2 for wavelengths ranging from 850 to $1070 \mathrm{~nm}$. For wavelengths of $\leqslant 800 \mathrm{~nm}$ we found that the bandwidth is limited by the electronics $(600 \mathrm{kHz})$. Above $800 \mathrm{~nm}$, silicon becomes increasingly transparent for increasing wavelengths. The output of the diode becomes effectively low-pass filtered $\left(f_{3 \mathrm{~dB}} \approx 10-12 \mathrm{kHz}\right.$ at $\left.1070 \mathrm{~nm}\right)$, where the attenuation is less than $6 \mathrm{~dB}$ /octave and strongly dependent on the wavelength. In Fig. 2 the solid line indicates the slope of a first-order low-pass filter ( $-6 \mathrm{~dB}$ /octave).

Applying a bias voltage will increase the thickness of the depletion layer of the silicon-based detector and therefore increase the bandwidth of the detector;

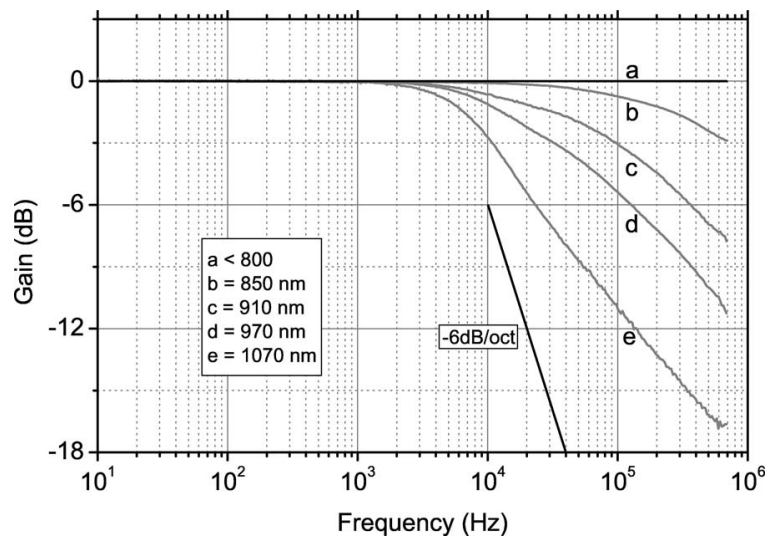

Fig. 2. Frequency response of a quadrant detector (S5891, Hamamatsu) operating at no bias for several wavelengths with a light power of $500 \mu \mathrm{W}$. Curves a-e show the frequency response for wavelengths of $\leqslant 800,850,910,970$, and $1070 \mathrm{~nm}$.

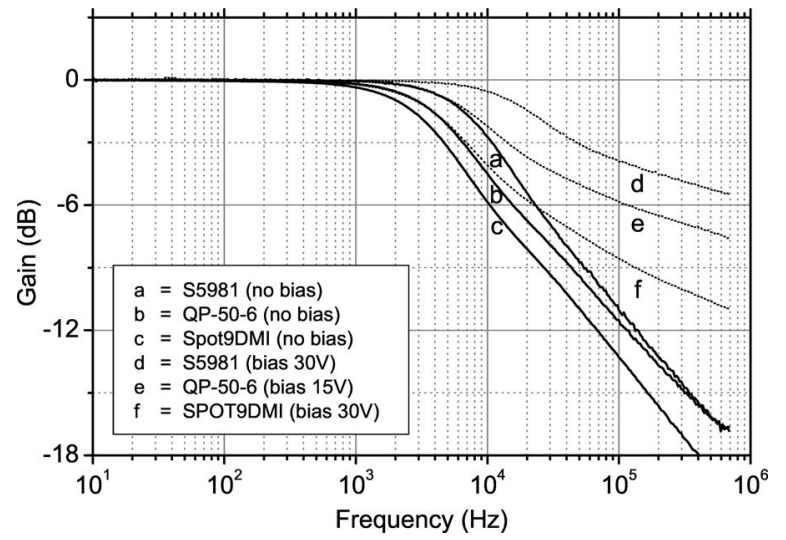

Fig. 3. Frequency response of the S5981 (Hamamatsu), the QP-50-6 (Pacific Silicon Sensor) and the SPOT9DMI (UDT) detectors operating with and without bias (all QDs), measured at a wavelength of $1070 \mathrm{~nm}$ and a light power of $500 \mu \mathrm{W}$. Curves a-c were acquired with no bias. Curves $\mathrm{d}-\mathrm{f}$ correspond to the same detectors but operating with bias (S5891, bias $30 \mathrm{~V}$; SPOT9DMI, bias $30 \mathrm{~V}$; QP-50-6, bias $15 \mathrm{~V})$.

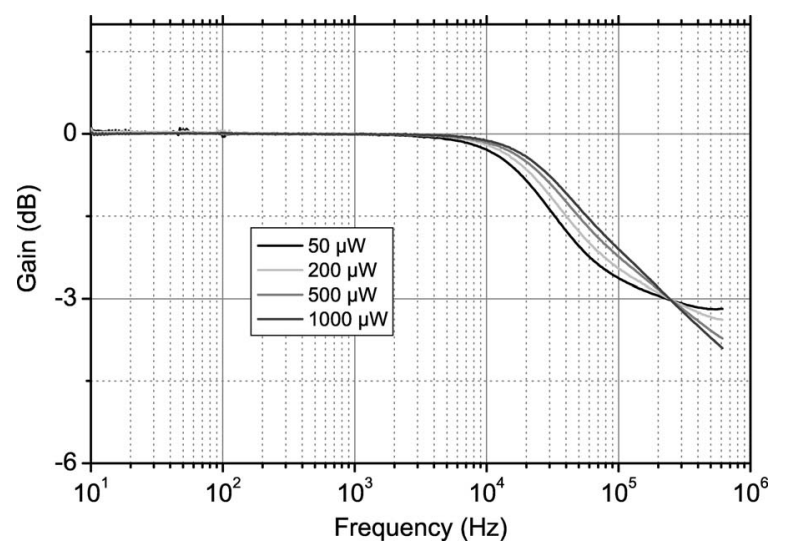

Fig. 4. Frequency response of the DL100-7-KER (Pacific Silicon Sensor) PSD for four total intensities (the wavelength used was $1070 \mathrm{~nm}$ ). Compare the vertical scale with those of the previous figures.

in Fig. 3 we demonstrate this by showing the frequency response of three QDs operating with and without a bias voltage. The curves are acquired at a wavelength of $1070 \mathrm{~nm}$ with a light power of $500 \mu \mathrm{W}$. All three detectors show an increase in bandwidth where a bias voltage is applied. Beyond $10 \mathrm{kHz}$, where all detectors already show the low-pass effect, a small nick in the curves is visible, after which the attenuation further exhibits a smaller slope.

The influence of the incident light power on the frequency response of the detectors is demonstrated for the DL100-7-KER PSD in Fig. 4. The responses were acquired at light powers of 50, 200, 500, and $1000 \mu \mathrm{W}$ at a wavelength of $1070 \mathrm{~nm}$. An increase in light power resulted in a decrease of the low-pass effect. For this detector we found that at a frequency near $230 \mathrm{kHz}$ the curves cross each other, after which a smaller attenuation was found at lower light powers with respect to higher powers. Other detectors showed a similar response to light power, and a crossing of the curves for different powers was observed. For detectors with a larger low-pass effect the cross- 
ing of the curves was found at lower frequencies. In Table 1 the bandwidth of the DL100-7-KER and the DLS10 (UDT) (both PSDs) and the SPOT9DMI (UDT; a QD) are given for light powers of 50 and $500 \mu \mathrm{W}$. For all detectors an increase in bandwidth is found for increasing light power. The DL100-7-KER detector exhibits a notably higher bandwidth with respect to the other detectors (shown previously by Neuman and Block. ${ }^{6}$ ) The effect of light power on the low-pass effect up to the crossing region suggests a saturation effect of the electron-hole pairs created outside the depletion layer caused by their relatively slow diffusion. If this effect indeed can be explained by saturation, it will be dependent on the total intensity flux and therefore also on the spot size, a factor that has not been investigated in this work.

Finally, we show in Fig. 5 the bandwidth as a function of wavelength for the various detectors used in the measurements at a light power of $500 \mu \mathrm{W}$ and at operating bias voltages indicated in the figure. At 850 $\mathrm{nm}$ the first detectors become bandwidth limited by the low-pass effect of the detector.

The LED wobbler described here is a simple, powerful, and low-cost tool with which to determine the frequency response of a detector with high accuracy for different wavelengths and light powers up to high operating frequencies, in our case limited by the bandwidth of the electronics $(600 \mathrm{kHz})$. As shown for QDs and PSDs, silicon-based detectors become bandwidth limited for wavelengths of $\geqslant 850 \mathrm{~nm}$ at which the low-pass effect is dependent on the detector, the applied bias voltage, and the light power. The frequency response of a detector thus obtained can be applied effectively to the correction of acquired power spectral densities in applications such as OT and

Table 1. Influence of Light Power on the Bandwidths of Detectors

\begin{tabular}{lcc}
\hline Detector & Light Power $(\mu \mathrm{W})$ & $f_{-3 d B}(\mathrm{kHz})$ \\
\hline DL100-7-KER & 50 & 230 \\
& 500 & 244 \\
DLS10 & 50 & 10.9 \\
& 500 & 15.4 \\
SPOT9DMI & 50 & 6.3 \\
& 500 & 6.9 \\
\hline
\end{tabular}

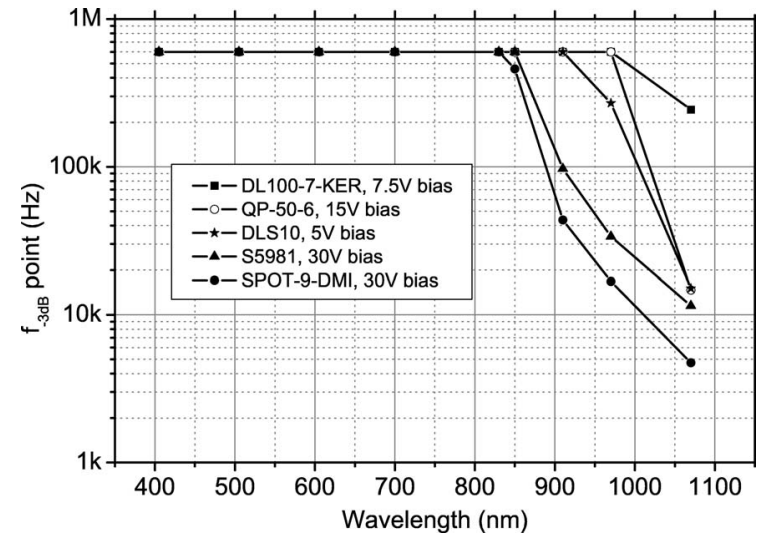

Fig. 5. Bandwidth as a function of wavelength for several detectors. The maximum bandwidth of $600 \mathrm{kHz}$ is the bandwidth of the electronics.

AFM. A detailed study for these key applications and of a first-order model describing the low-pass effect is in progress.

Alternative solutions to prevent unintended lowpass filtering include the use of an InGaAs detector (for near-infrared light), for which a potential drawback can be the size of the active area, currently $\leqslant 4 \mathrm{~mm}$, and the use of another or a second light source operating at a shorter wavelength $(<850 \mathrm{~nm})$.

We thank Pacific Silicon Sensor for providing us with its position detectors. This study is supported by the MESA+Institute. J. H. G. Huisstede's e-mail address is j.h.g.huisstede@utwente.nl.

\section{References}

1. G. Meyer and N. M. Amer, Appl. Phys. Lett. 53, 1045 (1988).

2. K. Svoboda and S. M. Block, Annu. Rev. Biophys. Biomol. Struct. 23, 247 (1994).

3. F. Gittes and C. F. Schmidt, Eur. Biophys. J. 27, 75 (1998).

4. K. Berg-Sørensen, L. Oddershede, E. L. Florin, and H. Flyvbjerg, J. Appl. Phys. 93, 3167 (2003).

5. E. J. G. Peterman, M. A. van Dijk, L. C. Kapitein, and C. F. Schmidt, Rev. Sci. Instrum. 74, 3246 (2003).

6. K. C. Neuman and S. M. Block, Rev. Sci. Instrum. 75, 2787 (2004).

7. J. H. G. Huisstede, K. O. van der Werf, M. L. Bennink, and V. Subramaniam, Opt. Express 13, 1113 (2005). 